\title{
REVIEW
}

\section{Biology of tooth replacement in amniotes}

\author{
John A Whitlock and Joy M Richman
}

Tooth replacement is a common trait to most vertebrates, including mammals. Mammals, however, have lost the capacity for continuous tooth renewal seen in most other vertebrates, and typically have only 1-2 generations of teeth. Here, we review the mechanisms of tooth replacement in reptiles and mammals, and discuss in detail the current and historical theories on control of timing and pattern of tooth replacement and development.

International Journal of Oral Science (2013) 5, 66-70; doi:10.1038/ijos.2013.36; published online 21 June 2013

Keywords: dental lamina; growth factor signalling; mammal; epithelial stem cells diphyodonty; polyphyodonty; reptile; successional lamina; tooth replacement; Zahnreihen, zone of inhibition

\section{INTRODUCTION}

The majority of vertebrates, including fish, sharks, amphibians and reptiles retain the capacity to renew their dentition multiple times throughout life (polyphyodonty). In contrast, most mammals can exchange their teeth only once (diphyodonty). ${ }^{1}$ Here we examine the reasons for these differences in tooth renewal competence. We compare the molecular controls of tooth replacement in the two branches of amniotes, mammals and reptiles. We also discuss the contribution of stem cells to the replacement process. Finally, we review the theoretical models of tooth replacement along the entire dentition and reconcile these with modern molecular insights.

\section{THE DENTAL AND SUCCESSIONAL LAMINAE}

As a prelude to discussing the details of tooth renewal, it is necessary to understand the dental epithelial structures that support this process. The dental lamina is the ingrowth of oral epithelium into the facial mesenchyme. The lamina is present in the embryonic facial prominences and is a multilayered tongue of epithelium which will give rise to the enamel organs (Figure $1 \mathrm{a}$ and $1 \mathrm{~b}$ ). Even though there is no tooth morphology present in the dental lamina, it is already patterned in three axes, labial-lingual, oral-aboral and anterior-posterior. The asymmetry is manifested in gene expression and cell proliferation patterns.

Beginning with the labial-lingual axis, there are restricted patterns of expression of several genes ${ }^{2-3}$ including sonic Hedgehog (Shh) which is expressed on the lingual side, Wingless-related proteins and Ectodysplasin receptor (Edar) which are also expressed on the labial side of the early dental lamina. ${ }^{4}$ These differences are correlated with higher proliferation on the labial side. Labial-lingual asymmetry may specify the tooth forming capabilities of the labial side of the lamina. In mammals, there are also labial-lingual differences in gene expression in the dental lamina of ferrets (Sclerostin domain-containing protein 1, Wnt and Bmp antagonist, Sostdc1). ${ }^{5}$
The second axis is the oral-aboral axis. In this plane, the dental lamina connects the oral epithelium to the different generations of teeth (Figure 1c). At the free end of the dental lamina, there is usually higher cell proliferation and higher expression of the Wnt target gene, Lef1. ${ }^{2}$ The growing tip becomes the successional lamina when it extends off the most immature tooth in a tooth family. Setting aside cells that will form the successional lamina, over and over again is critically important for polyphyodonty. We have hypothesized that in mammals the absence of a third generation of teeth could be due to the failure to form a successional lamina from the permanent tooth buds or that a successional lamina forms, but it regresses prior to forming a tooth. Evidence of a 'third generation' successional lamina in human was reported in one study. ${ }^{6}$ In this detailed analysis of the human dentition, the mandibular permanent lateral incisors had an epithelial lamina originating from the enamel organ which is a position where supernumerary teeth often form. ${ }^{7}$ Thus, the potential to form supernumerary teeth may correlate with the presence of a vestigial third generation successional lamina in some regions of the mouth. In mammals, the dental lamina connecting the primary tooth and the oral epithelium degrades ${ }^{6,8-9}$ (Figure 1d), and eventually, the primary tooth erupts into the oral cavity (Figure 1d). This degeneration of the dental lamina has been suggested to be due a combination of epithelialto-mesenchymal transformation and possibly apoptosis in the minipig model. ${ }^{8}$ This same group of investigators have proposed that the loss of the connection between the dental lamina and oral cavity in mammals may be related to the inability to form a third or subsequent generations of teeth. ${ }^{8}$ In many reptiles, there is a robust connection between tooth generations that is never lost even into adult hood ${ }^{10}$ (Figure 1c).

To explore the idea of a vestigial successional lamina further, we investigated a reptile that only forms 2 sets of teeth, the bearded dragon. Indeed in prehatching animals we found a short, thin, rudimentary successional lamina. We were unable to detect expression Axin2 or Tcf7 (transcription factor 7, another Wnt pathway transcription factor and 
a

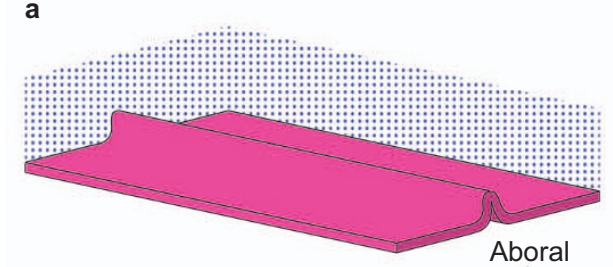

Early dental lamina

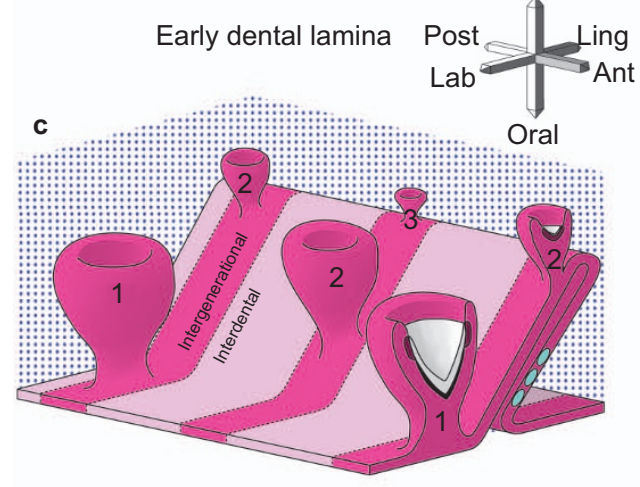

Reptilian tooth families b

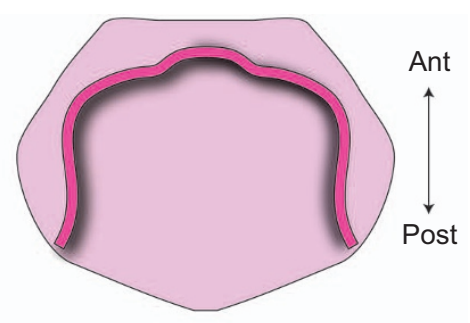

Continuous dental lamina around maxillary arch

d

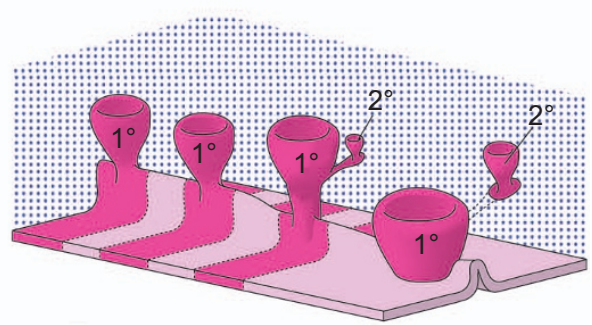

Mammalian tooth development and succession

Figure 1 Development of generational teeth in a diphyodont mammal and a polyphyodont reptile. (a, b) The early dental lamina in both reptiles and at least some mammals, including humans, is a continuous invagination of oral epithelium (pink tones) into the dental mesenchyme (blue stippling) that will give rise to the all teeth of the primary dentition. $\mathbf{b}$ is redrawn from ref. 6. (c) In reptiles, the dental lamina remains continuous in both the intergenerational (dark pink) and interdental (lighter pink) regions. Tooth families contain several generations of teeth $(1,2,3)$ at progressive stages of development. The lingual side or non-tooth forming side of the dental lamina contains the label-retaining putative stem cells (green circles). The successional lamina continues as an extension of the dental lamina off the newest forming tooth. (d) In diphyodont mammals, the dental lamina is continuous and connects adjacent teeth of the primary dentition until the dental lamina degrades in the bell stage, disconnecting the enamel organ from the oral epithelium. The permanent tooth bud is also disconnected from the primary tooth once the primary tooth begins root formation. Three-axis compass rose orients $\mathbf{a}, \mathbf{c}$ and $\mathbf{d}$; single axis rose orients $\mathbf{b}$.

target gene) in the rudimentary successional lamina. ${ }^{4}$ The lack of Wnt readouts in bearded dragon contrasts the high Wnt activity in the snake successional lamina. ${ }^{2}$ Ultimately, increased apoptosis occurs in the bearded dragon teeth which we predicted would result in loss of the successional lamina some time during the post-hatching period. This prediction was recently tested in chameleons that only form a single generation of teeth. ${ }^{11}$ These authors observed a successional lamina in pre- and post-hatching juveniles, although the relative size of the lamina is much smaller in the older animals. These size differences correlate with reduced proliferation in the juvenile successional lamina. Thus, for the first time, the mechanism for loss of tooth capacity in adults can be directly attributed to lack of growth of the rudimentary successional lamina.

The third axis of the dental lamina is the anterior-posterior axis. It may be surprising to learn that the primary dental lamina of humans most closely resembles that of the reptile and not that of the mouse. Examination of serial reconstructions of embryonic and foetal human jaws carried out by Ooë ${ }^{6}$ leaves no doubt that a continuous primary dental lamina is present around the jaw in the late embryonic and fetal periods (schematized in Figure 1b). The primary teeth form within this dental lamina and remain connected to it until the roots begin to form or late bell stage. In addition reconstructions of the ferret dental lamina report similar continuity between teeth of the primary dentition. ${ }^{5}$ The appearance of this primary mammalian dental lamina is virtually indistinguishable from the snakes and geckos that we have reconstructed (Figure $1 \mathrm{c}$ and $1 \mathrm{~d}$ ). ${ }^{10,12}$ However, unlike mammals, the continuous dental lamina surrounding the jaw in reptiles persists into post-hatching animals. ${ }^{10}$

In rodents, there does not appear to be a continuous dental lamina. Instead the odontogenic band which consists of a region of localized gene expression marking the position of the tooth row is resolved into discontinuous placodes, one for the continuously erupting incisors and one for the molars which are never replaced. ${ }^{13}$ There is a diastema between the incisor and molar placode in mice where vestigial tooth buds form and regress. ${ }^{14}$ Thus the lack of a continuous dental lamina in rodents is a part of their specialized dentition. The one place in the rodent dentition where the dental lamina extends is in the molar region. ${ }^{6,13}$ The contribution of the dental lamina of the first molar to the addition of second and third molars has been recently proven in a fate-mapping experiment. The authors took advantage of the Sox2 transcription factor which is expressed strongly in the dental lamina of mice, ferrets, snakes, lizards and alligators. ${ }^{15}$ In the mouse model, a Sox2-CreERT2 line was crossed with a Rosa26-reporter line (Sox2CreERT2;R26R ${ }^{\text {lacZ }}$ ) and expression was turned on by injection of Tamoxifen at E13.0. ${ }^{15}$ It was possible to show in culture that second and third molar enamel organs derive from the Sox2-expressing dental lamina. Taking the fate mapping data together with the conserved Sox2 expression in mammals and reptiles, we now believe that we can use the addition of mouse molars to understand some of the molecular mechanisms underpinning tooth renewal.

In humans, non-rodent mammals and reptiles, it is not clear what positive or negative signals may act to restrict tooth formation to defined locations along the continuous dental lamina. For example, careful examination of the diphyodont shrew and ferret have not yet discovered a gene coding for an activator or inhibitor that is regionally restricted in the dental lamina. ${ }^{5,16}$ Examples of activators are the Wnt ligands and inhibitors of this pathway include secreted frizzled-related (Sfrp), Dikkopf (Dkk) or Sostdc proteins. So far in reptiles our regional analysis of gene expression is limited to serial sections 
through a section of snake dental lamina probed for the activator, Shh. ${ }^{12}$ However, here we found continuous expression of Shh in the oral part of the dental lamina with no interruption along the anteriorposterior axis. Shh is also expressed in the inner enamel epithelium of the tooth once it has formed. We exclude Shh as being a periodic activator in the dental lamina; however, more three-dimensional reconstructions of gene expression are needed in both reptiles and diphyodont mammals to identify putative dental patterning signals.

\section{ROLE OF EPITHELIAL STEM CELLS IN TOOTH REPLACEMENT}

Repeated organogenesis requires a store of pleuripotent cells to enable growth of new tissues; in other ectodermal organ systems, such as hair and continuously erupting mouse incisors, stem cells have been identified. ${ }^{17-26}$ Recent studies have used GFP driven by the Sox2 promoter to identify stem cells in the labial incisor cervical loop. ${ }^{27}$ These cells give rise to all of the labial ameloblasts and are responsible for continued growth. It is interesting that in the incisor and hair there are recognizable thickenings that contain the stem cells. The slowly dividing stem cells replace themselves and are a stable population that can always be seen in the niche. We have looked for a thickened region of the dental epithelium in reptilian dentitions but have not found such structures. Instead, we used a labeling method adapted from hair research to mark the most slowly dividing cells in the dental epithelium and by proxy, mark the putative stem cells. ${ }^{10}$ Label retaining cells were clustered in groups, generally on the lingual surface of the intergenerational dental lamina (Figure 1c). These label retaining cells are Wnt-responsive and express several stem cell markers such as Lgr5, $D k k 3$ and Igfbp5. These label-retaining cells remain in this position over a protracted period of time, up to 5 months, during which teeth would have been exchanged 2-3 times. We hypothesize that this population of label retaining cells forms a self-replenishing population in between tooth families that is regulated by a stationary niche in the adjacent mesenchyme. ${ }^{4,28}$ Future work will focus on identifying the niche and tracing the fate of the label retaining cells in order to see whether they do indeed give rise to all parts of the enamel organ and successional lamina.

\section{JAW-WIDE PATTERNS OF TOOTH REPLACEMENT}

The replacement of teeth, in all animals for which it occurs, occurs on regular schedules that vary between animals and are not related directly to wear, breakage or loss of teeth. ${ }^{29-30}$ In humans, the sequence of permanent tooth formation and eruption is not linear but instead is more advanced in the incisor region compared the premolar with the maxillary canines being the last to be replaced. ${ }^{31}$ Patterns of tooth replacement are also seen in reptiles, but here instead of being regionalized to certain parts of the jaw, there is generally an alternating sequence. Previous work stretching back almost 100 years on pre- and post-hatching crocodiles, ${ }^{32}$ chameleons ${ }^{33}$ and tuataras ${ }^{34}$ found that positions 1, 3, 5 et al. are most similar, in size and stage of development, as are teeth in positions 2, 4, 6 et al. (Figure 2). Based on these data, several scientists have concluded that teeth at different positions along the jaw initiate independently. ${ }^{30,34}$ Woerdeman $^{35}$ was the first person to propose that teeth arise and replace in waves and coined the term Zahnreihen to describe this pattern. In its purest sense, a Zahnreihe consists of a group of neighboring (adjacent) teeth from different tooth families that are at a similar stage of development (anterior to posterior diagonal lines, Figure 2). The diagonal arrangement of the Zahnreihe is superimposed on the straight line of replacement teeth within single-tooth families. In this way, should several teeth be exfoliated at the same time, there would be some teeth close to

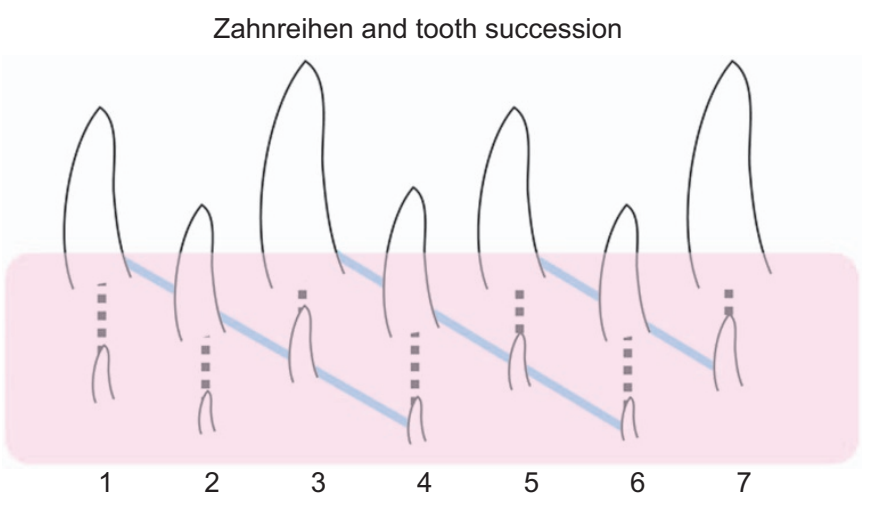

Figure 2 Diagrammatic representation of the first seven tooth families in a hypothetical reptile jaw. Diagonal lines connecting teeth in decreasing stages of development, or Zahnreihen, can be drawn across tooth families (shown in blue), illustrating the apparent 'waves' of tooth replacement. The dashed line shows succession within a single tooth family. The pink layer indicates the teeth that are not visible in the oral cavity and are still forming. Consequently, Zahnreihen can only be seen on radiographs or in histology.

being fully formed and ready to erupt to fill the gap. In the human dentition during transition from the primary to permanent dentition, there is also staggered development so that all incisors or deciduous molars are not exfoliated at the same time as their nearest neighbours.

\section{Theories on the control of replacement - wave stimulus and zone of} inhibition

Well before the start of the molecular age, Edmund ${ }^{30,36-37}$ proposed a theory to explain the alternating patterns of tooth replacement. $\mathrm{He}$ suggested that an extrinsic 'wave stimulus' originates in the anterior dental mesenchyme and is transmitted posteriorly through the odontogenic anlage, stimulating tooth growth as it passes. The stimulus or signal would diminish in intensity leaving the intervening tooth at a more immature stage. Subsequent wave stimuli appear along the jaw and are responsible for subsequent generations of teeth; temporal staggering of these stimuli sets up the pattern of replacement observed (Figure $3 \mathrm{a}$ and $3 \mathrm{a}^{\prime}$ ). This hypothesis was predicated on two assumptions common at the time: (i) the first tooth to develop is typically the first/most mesial; and (ii) teeth are progressively initiated in order along the length of the tooth row. Although these ideas were initially widely accepted (e.g. refs. 38-40, subsequent work on lizards and alligators ${ }^{41-44}$ has shown that neither assumption is correct. Recent chameleon data nicely shows that areas of tooth initiation are not orderly in the anterior-posterior direction but instead seem to correlate with regions of increased jaw growth. ${ }^{11}$ In other words, when space is available, additional teeth form.

The Zahnreihen break down with increasing maturity of the individual $^{30,45}$ and at different positions around the dental arcade. ${ }^{38,46-47}$ Replacement waves even appear to reverse at various points in the jaw. ${ }^{47}$ Regional differences in replacement capacity also exist, with replacement occurring anteriorly, but not at all in the distal-most dentition in some lizards. ${ }^{38}$ Taken together, the observed variability in dental replacement patterns illustrate that it may be hard to generalize. Furthermore, these natural variations between reptiles highlight the necessity of getting to know your animal model in detail before embarking on experimental perturbations.

The second model to explain Zahnreihen is the 'zone of inhibition' (ZOI) theory, which suggests that a developing tooth emits a signal into the surrounding mesenchyme that inhibits the development of 
a

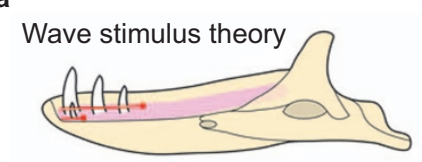

$a^{\prime}$

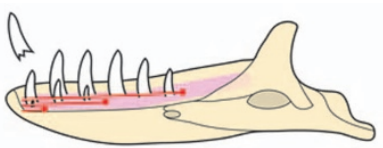

b Zone of inhibition theory

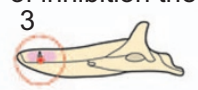

b"

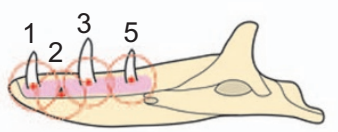

b

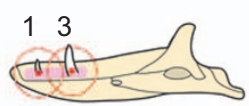

b"'

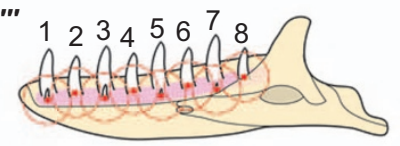

Figure 3 Schematic of the two main theories controlling the pattern of tooth replacement observed in reptiles. (a-a') Edmund's 'wave stimulus', an extrinsic chemical signal, originates from near the first tooth position and disperses distally along the odontogenic anlage, inducing the initiation of tooth development as it proceeds. In a, the first wave stimulus has just passed tooth position 3, resulting in the development of the first three teeth. The second wave stimulus has just passed tooth position 1 , initiating a second wave of tooth development. In $\mathbf{a}^{\prime}$, the first two wave stimuli have continued posteriorly, and a third stimulus has just passed the first tooth position, where an erupted tooth has just been shed. (b$\mathbf{b}^{\prime \prime \prime \prime}$ ) Osborn's 'zone of inhibition' theory. In b, the first tooth to develop (future tooth position 3) generates an as-yet-unknown signal inhibiting tooth development (red dot) diffusing out in all directions. As the odontogenic anlage is still small, the secreted molecule inhibits tooth development throughout the entire structure. $\mathbf{b}^{\prime}-\mathbf{b}^{\prime \prime}$ " illustrate how growth influences the physical spacing of teeth and thereby the distance between ZOI. ZOI, zone of inhibition.

additional teeth within a certain radius. ${ }^{48-49}$ Under this hypothesis, the dental lamina is a constantly proliferative area of potential tooth forming cells. Initiation of each new tooth enlarges the ZOI, preventing uncontrolled tooth formation (Figure $3 b-3 b^{\prime \prime \prime}$ ). As teeth erupt and as the jaw grows anterior-posteriorly, space is created for the initiation of additional teeth. Assuming the ZOI is roughly spherical (as would result from a secreted inhibitor diffusing equally from a central point), once the tooth expressing the inhibitor has erupted, the dental lamina on either side is no longer inhibited and thus is free to develop teeth. In this way, an alternating pattern is constructed, based entirely on the order of initiation.

Much as we have discussed, the activators or inhibitors that could set up the ZOIs have not yet been identified. However, there are several candidate pathways that have been shown to play a role in spacing of other ectodermal organs, such as feathers. ${ }^{50-53}$ A reaction-diffusion reaction prevents the initiation of a replacement structure until the functional structure has reached a certain stage of maturity. In particular, Wnt, Fgf, Eda and Bmp pathways are among those that have been implicated as potential regulators of feather spacing. ${ }^{52-55}$ Our lab has mapped expression of a Wnt antagonist, Secreted frizzled-related protein (Sfrp2) in pythons and bearded dragons (Handrigan and Richman, unpublished data). We found that it was expressed around the tooth anlagen but not within the teeth themselves. This pattern is complementary to areas of high Wnt activity within and next to the successional lamina and suggests that $S f r p 2$ may help to restrict tooth formation to odontogenic mesenchyme.

The best functional evidence for an inhibitory mechanism in facial mesenchyme comes from an organ culture experiment using mouse incisor tooth germs. By trimming the mesenchyme around the incisors very close to the teeth, supernumerary teeth formed. ${ }^{56}$ This demonstrated that inhibitory factors in the mesenchyme limited the number

of teeth. One such molecule is the Bmp and Wnt antagonist, Sostdc1, which is expressed in the mesenchyme. Indeed, the deletion of Sostdc1 in epithelium and mesenchyme leads to formation of double incisors. ${ }^{57}$ Therefore, increased Bmp and Wnt activity leads to deregulation of tooth number. However, the tissue in which gene inactivation occurs is critical. In other studies, disrupting Wnt signalling specifically in the mesenchyme ${ }^{58}$ leads to a downregulation of Bmp4. Although these gene expression changes are opposite to the Sostdc1 mutant animals, supernumerary incisors also form. These seemingly contradictory results demonstrate that there is a dialogue between epithelium and mesenchyme which regulates tooth number. Finally, in a different approach, inhibitors were placed directly into the incisor mesenchyme, essentially creating an ectopic ZOI very close to a tooth bud. The high level of inhibitor caused the single tooth germ to split up into several incisors in organ culture. ${ }^{56}$ Based on this mouse data, the mesenchyme is important in negatively regulating tooth number and that ZOI may be created by spatially restricted expression of antagonists such as those that inhibit Wnt activators. The advantage of such zones of inhibition in the human dentition would be that crowding of teeth would be prevented. Teeth that are too close together during development may increase the probability of defects in either the crown or root. By delaying development of the terminal tooth in each tooth morphotype (incisor, premolar, molar) jaw size can increase and there will be room for all the permanent dentition.

\section{CONCLUSIONS}

The transition from polyphyodonty in extinct mammals to diphyodonty in modern mammals may be due to (i) the loss of integrity of the dental lamina which in turn leads to loss of dental epithelial stem cells; (ii) inability to reform a successional lamina which also could be due to loss of the stem cell niche; or (iii) vestigialization of a successional lamina. The reptile and mouse molars are both good models to explore the molecular underpinnings of tooth renewal. The reptile model will give additional insights into the patterning of tooth renewal along the jaw including whether there are inhibitory signals prevent initiation of a tooth within a certain radius of the existing tooth.

\section{ACKNOWLEDGEMENTS}

Some of this work was presented at the West China School of Stomatology, Chengdu, China during the 2012 International Symposium on Tooth development. The authors would like to thank Dr Jian (Jerry) Q Feng and colleagues at the State Key Laboratory of Oral Disease for organizing this conference. The reptile studies were funded by an NSERC grant to Joy M Richman. Publication of this manuscript is supported by Open Fund of State Key Laboratory of Oral Diseases, Sichuan University.

1 Jernvall J, Thesleff I. Tooth shape formation and tooth renewal: evolving with the same signals. Development 2012; 139(19): 3487-3497.

2 Handrigan GR, Richman JM. A network of Wnt, hedgehog and BMP signalling pathways regulates tooth replacement in snakes. Dev Biol 2010; 348(1): 130-141.

3 Handrigan GR, Richman JM. Autocrine and paracrine Shh signaling are necessary for tooth morphogenesis, but not tooth replacement in snakes and lizards (Squamata). Dev Biol 2010; 337(1): 171-186.

4 Richman JM, Handrigan GR. Reptilian tooth development. Genesis 2011; 49(4): 247-260.

5 Järvinen $E$, Tummers $M$, Thesleff $I$. The role of the dental lamina in mammalian tooth replacement. J Exp Zool B Mol Dev Evol 2009; 312B(4): 281-291.

6 Ooë T. Human Tooth and Dental Arch Development. Tokyo: Ishiyaku Publishers Inc., 1981: 217.

7 Wang XP, Fan J. Molecular genetics of supernumerary tooth formation. Genesis 2011; 49(4): 261-277

8 Buchtová M, Stembirek J, Glocova K et al. Early regression of the dental lamina underlies the development of diphyodont dentitions. J Dent Res 2012; 91(5): 491498. 
9 Štembírek J, Buchtová M, Král T et al. Early morphogenesis of heterodont dentition in minipigs. Eur J Oral Sci 2010; 118(6): 547-558.

10 Handrigan GR, Leung KJ, Richman JM. Identification of putative dental epithelial stem cells in a lizard with life-long tooth replacement. Development 2010; 137(21): 3545-3549.

11 Buchtová $\mathrm{M}$, Zahradnicek $\mathrm{O}$, Balkova S et al. Odontogenesis in the veiled Chameleon (Chamaeleo calyptratus). Arch Oral Biol 2012; pii: S0003-9969(12)00380-9. doi: 10.1016/j.archoralbio.2012.10.019. [Epub ahead of print].

12 Buchtová M, Handrigan G, Tucker A et al. Initiation and patterning of the snake dentition are dependent on Sonic Hedgehog signaling. Dev Biol 2008; 319(1): 132-145.

13 Jussila M, Thesleff I. Signaling networks regulating tooth organogenesis and regeneration, and the specification of dental mesenchymal and epithelial cell lineages. Cold Spring Harb Perspect Biol 2012; 4(4): a008425.

14 Peterkova R, Lesot H, Peterka M. Phylogenetic memory of developing mammalian dentition. J Exp Zool B Mol Dev Evol 2006; 306(3): 234-250.

15 Juuri E, Jussila M, Seidel K et al. Sox2 marks epithelial competence to generate teeth. Development 2013; 140(7): 1424-1432.

16 Järvinen E, Välimäki K, Pummila M et al. The taming of the shrew milk teeth. Evol Dev 2008; 10(4): 477-486

17 Chuong C, Wu P, Plikus M et al. Engineering stem cells into organs: topobiological transformations demonstrated by beak, feather, and other ectodermal organ morphogenesis. Curr Top Dev Biol 2006; 72: 237-274.

18 Cotsarelis G, Sun TT, Lavker RM. Label-retaining cells reside in the bulge area of pilosebaceous unit: implications for follicular stem cells, hair cycle, and skin carcinogenesis. Cel/ 1990; 61(7): 1329-1337.

19 Blanpain C, Fuchs E. Epidermal homeostasis: a balancing act of stem cells in the skin. Nat Rev Mol Cell Biol 2009; 10(3): 207-217.

20 Fuchs E, Horsley V. More than one way to skin.... Genes Dev 2008; 22(8): 976-985.

21 Tumbar T, Guasch G, Greco V et al. Defining the epithelial stem cell niche in skin. Science 2004; 303(5656): 359-363.

22 Harada $\mathrm{H}$, Kettunen $\mathrm{P}$, Jung $\mathrm{HS}$ et al. Localization of putative stem cells in dental epithelium and their association with Notch and FGF signaling. J Cell Biol 1999; 147(1): 105-120.

23 Harada H, Toyono T, Toyoshima $\mathrm{K}$ et al. FGF10 maintains stem cell population during mouse incisor development. Connect Tissue Res 2002; 43(2/3): 201-204.

24 Klein OD, Lyons DB, Balooch G et al. An FGF signaling loop sustains the generation of differentiated progeny from stem cells in mouse incisors. Development2008; 135(2): 377-385.

25 Thesleff I, Wang XP, Suomalainen M. Regulation of epithelial stem cells in tooth regeneration. CR Biol 2007; 330(6/7): 561-564.

26 Wang XP, Suomalainen M, Felszeghy S et al. An integrated gene regulatory network controls stem cell proliferation in teeth. PLoS Biol 2007; 5(6): e159.

27 Juuri E, Saito K, Ahtiainen L et al. Sox $2^{+}$stem cells contribute to all epithelial lineages of the tooth via Sfrp5 ${ }^{+}$progenitors. Dev Cell 2012; 23(2): 317-328.

28 Richman JM, Whitlock JA, Abramyan J. Reptilian tooth regeneration // Huang GTJ, Thesleff I. Stem cells in craniofacial development and regeneration. New York: WileyBlackwell, John Wiley \& Sons, 2013: 135-151.

29 Berkovitz BK. Tooth replacement patterns in non-mammalian vertebrates // Teaford FT, Smith MM, Ferguson MW. Development, function and evolution of teeth. Cambridge: Cambridge University Press, 2000: 186-200.

30 Edmund AG. Tooth replacement phenomena in the lower vertebrates. Contrib Life Sci Div R Ont Mus 1960; 52: 52-190.

31 Logan WH, Kronfeld R. Development of the human jaws and surrounding structures from birth to the age of fifteen years. J Am Dent Assoc 1933; 20: 379-427.

32 Röse C. [Über die Zahnentwicklung der Krokodile.] Morphol Jahrb 1893; 3(2): 195228. German.

33 Röse C. Ueber die Zahnentwicklung von Chamaeleon. Anat Anz 1893; 9: 439-451.

34 Harrison HS. The development and succession of teeth in Hatteria punctata. $Q \mathrm{~J}$ Microsc Sci 1901; 44: 161-219.

35 Woerdeman MW. [Beitrage zur Entwicklungsgeschichte von Zähnen und Gebiss der Reptilien. Beitrage IVUeber die Anlage des Ersatzgebiss.] Arch Mikrosk Anat 1921; 95(1): 265-395. German.
36 Edmund AG. Sequence and rate of tooth replacement in the Crocodilia. Contrib Life Sci Div R Ont Mus Toronto 1962; 56: 7-42.

37 Edmund AG. Dentition // Gans C, Bellaris A, Parsons T. Biology of the Reptilia. Morphology A. London: Academic Press, 1969: 117-200.

38 Cooper JS, Poole DF, Lawson R. The dentition of agamid lizards with special reference to tooth replacement. J Zool 1970; 162: 85-98.

39 Hopson JA. Postcanine replacement in the gomphodont cynodont Diademodon /I Kermack DM, Kermack KA. Early Mammals. London: Academic Press, 1971: 1-21.

40 Ziegler AC. A theory of the evolution of therian detal formulas and replacement patterns. Q Rev Biol 1971; 46: 226-249.

41 Osborn JW. New approach to Zahnreihen. Nature 1970; 225(5230): 343-346.

42 Osborn JW. The interpretation of patterns in dentition. Biol J Linnean Soc 1977; 9 : 217-229.

43 Westergaard B, Ferguson WJ. Development of the dentition in Alligator mississippiensis. Later development in the lower jaws of embryos, hatchlings and young juveniles. J Zoo 1987; 212: 191-222.

44 Westergaard B, Ferguson MW. Development of the dentition in Alligator mississippiensis: upper jaw dental and craniofacial development in embryos, hatchlings, and young juveniles, with a comparison to lower jaw development. Am J Anat 1990; 187(4): 393-421.

45 Miller WA, Radnor CJ. Tooth replacement patterns in young Caimen sclerops. J Morphol 1970; 130: 501-510.

46 Evans SE. Tooth replacement in the lower Jurassic lepidosaur Gephyrosaurus bridensis. N Jb Geol Paläont- Monat 1985; 7: 411-420.

47 Cooper JS. The dental anatomy of the genus Lacerta. Bristol: University of Bristol Press, 1963.

48 Osborn JW. The ontogeny of tooth succession in Lacerta vivipara Jacquin (1787). Proc $R$ Soc Lond B Biol Sci 1971; 179(56): 261-289.

49 Osborn JW. The evolution of dentitions. Am Scientist 1973; 61: 548-559.

50 Clauss F, Maniere MC, Obry F et al. Dento-craniofacial phenotypes and underlying molecular mechanisms in hypohidrotic ectodermal dysplasia (HED): a review. J Dent Res 2008; 87(12): 1089-1099.

51 Patel K, Makarenkova H, Jung HS. The role of long range, local and direct signalling molecules during chick feather bud development involving the BMPs, follistatin and the Eph receptor tyrosine kinase Eph-A4. Mech Dev 1999; 86(1/2): 51-62.

52 Chang $\mathrm{CH}$, Jiang TX, Lin CM et al. Distinct Wnt members regulate the hierarchical morphogenesis of skin regions (spinal tract) and individual feathers. Mech Dev2004, 121(2): 157-171

53 Widelitz RB, Jiang TX, Chen CW et al. Wnt-7a in feather morphogenesis: involvement of anterior-posterior asymmetry and proximal-distal elongation demonstrated with an in vitro reconstitution model. Development 1999; 126(12): 2577-2587.

54 Drew CF, Lin CM, Jiang TX et al. The Edar subfamily in feather placode formation. Dev Biol 2007; 305(1): 232-245.

55 Lin C, Jiang T, Baker R et al. Spots and stripes: pleomorphic patterning of stem cells via $\mathrm{p}$-ERK-dependent cell chemotaxis shown by feather morphogenesis and mathematical simulation. Dev Biol 2009; 334(2): 369-382.

56 Munne PM, Felszeghy S, Jussila M et al. Splitting placodes: effects of bone morphogenetic protein and activin on the patterning and identity of mouse incisors. Evol Dev 2010; 12(4): 383-392.

57 Munne PM, Tummers M, Järvinen E et al. Tinkering with the inductive mesenchyme Sostdc1 uncovers the role of dental mesenchyme in limiting tooth induction. Development 2009; 136(3): 393-402.

58 Fujimori S, Novak $\mathrm{H}$, Weissenbock $\mathrm{M}$ et al. Wnt/beta-catenin signaling in the dental mesenchyme regulates incisor development by regulating Bmp4. Dev Biol 2010 348(1): 97-106.

This work is licensed under a Creative Commons Attribution-NonCommercial-NoDerivative Works 3.0 Unported License. To view a copy of this license, visit http:// creativecommons.org/licenses/by-nc-nd/3.0 